\title{
Movilizando la subalternidad: la sindicalización de trabajadoras subcontratadas de aseo en una universidad privada chilena.
}

\author{
Mobilizing subalternity: outsourced cleaning-workers' unionization \\ in a Chilean university.
}

Fernanda Rojas Müller ${ }^{*}$

\section{Resumen}

Este artículo examina las posibilidades de organización de trabajadoras subcontratadas de aseo a partir de un estudio de caso con una delegación sindical de una universidad privada en Chile. Debido a las condiciones precarias del trabajo subcontratado, estos trabajadores han sido caracterizados como difíciles de organizar y movilizar. No obstante, en 2017 un grupo de trabajadoras de aseo se unió a un sindicato interempresa nacional y en 2018 llevó a cabo un proceso de huelga laboral. Este artículo propone que la intersección de opresiones de raza, género y clase en el marco de un trabajo reproductivo precarizado, sumados al impacto de una gubernamentalidad neoliberal configura a estas trabajadoras como subalternas en el sentido gramsciano. La investigación concluye que la sindicalización de estas trabajadoras fue posible precisamente porque movilizaron su subalternidad y que el éxito de sus movilizaciones responde a que se involucraron en formas de sindicalismo comunitario.

Palabras clave: trabajo subcontratado de aseo, interseccionalidad, nuevo sindicalismo, subalternidad, neoliberalismo

\begin{abstract}
This article investigates outsourced cleaning-workers' possibilities of mobilization through a case study focused on a group of workers in a Chilean private university. Outsourced cleaning services within universities are characterized by their precarious working conditions and their workers have been therefore portrayed as harder to organize and mobilize. However, in 2017, eight outsourced cleaning-workers joined a national intercompany union and in 2018 called a strike, constituting themselves as the first outsourced cleaning workers' union to achieve that within the university's history. I argue that their precarious working condition under neoliberalism combined with intersections of class, gender, and race oppression configure the workers as subalterns in the Gramscian sense. Furthermore, I claim that subalternity fostered their mobilization and its success was due to their engagement in local forms of community unionism.
\end{abstract}

\footnotetext{
* Licenciada en Letras Pontificia Universidad Católica de Chile, Magister en Género, London School of Economics and Political Science
} 
Key words: outsourced cleaning work, intersectionality, new syndicalism, subalternity, neoliberalism

Fecha de recepción: Noviembre 2020

Fecha de aprobación: Junio 2021

\section{Introducción}

En junio de 2017, ocho trabajadoras subcontratadas ${ }^{1}$ de aseo de una universidad privada en Chile formaron una delegación sindical. Un año después, dicha delegación tenía 25 socias que realizaron una negociación colectiva y posterior huelga laboral para mejorar sus condiciones de trabajo y sus salarios. Según las trabajadoras, tanto la huelga como los otros logros de la delegación sindical son excepcionales en la historia de las trabajadoras subcontratadas de aseo de dicha universidad. Para ellas, todo el proceso fue un "mito" que nunca pensaron iban a experimentar, mucho menos, liderar. Su percepción es reafirmada por académicos que han descrito los servicios subcontratados de aseo en Chile (Boccardo, Cornejo, Gómez-Rubio, Vidal, \& Zavala-Villalón, 2015; Boccardo, Moya, Ratto, \& Ruiz, 2016; Fundación Sol, 2014) y por otros campos en que las trabajadoras de aseo subcontratadas han sido consideradas difíciles de organizar por sus condiciones de vida y trabajo (Alberti, 2016).

Sin embargo, las trabajadoras de aseo se han movilizado contra el neoliberalismo y han demandado mejoras en sus condiciones laborales y más desde los años 70 en el Reino Unido y Estados Unidos (Rowbotham, 2006; Herod \& Aguiar, 2006b), y en otros contextos (Hearn \& Bergos, 2011; Soni - Sinha \& Yates, 2013; Van Vlijmen, 2019; Wills, 2008) que se han visto enfrentados al debilitamiento del sindicalismo, a la flexibilización del mercado del trabajo y la consecuente externalización de diversas áreas (Harvey, 2007a). De hecho, se ha considerado a estas trabajadoras como una vanguardia poco esperable y probable en el marco de un movimiento laboral urbano transnacional (Wills, 2008). En Chile hay ejemplos de sindicalismo y movilizaciones de trabajadores subcontratados sin precedentes en áreas como la minería, la industria del salmón y las forestales (Echeverría, 2010, 2013; Ruiz \& Boccardo, 2014) y de sindicalización femenina en trabajos en condiciones precarias y/o subcontratadas en otras áreas que no son los servicios de aseo (Cid, 2012; Valdés, Godoy, \& Mendoza, 2017).

En este sentido, siguiendo a Acciari (2018), en este artículo planteo que, más que ausencia de procesos de organización de trabajadoras subcontratadas de aseo, probablemente la academia no les ha prestado suficiente atención. Esto no es una crítica a aquellas investigaciones que se han enfocado en trabajadoras y un tipo de trabajo que es altamente invisibilizado e ignorado (Aguiar, 2004), por el contrario, el llamado es a seguir haciéndolo, abordando particularmente sus formas de organización (sindicalización

\footnotetext{
${ }^{1}$ A lo largo de este artículo me referiré en femenino a las trabajadoras subcontratadas de aseo en tanto, por lo menos en universidades de Santiago, son en su mayoría mujeres (Boccardo et al., 2015; Boccardo, et al., 2016; Fundación Sol, 2014).
} 
y otras). Ese es precisamente el objetivo y contribución de este artículo ${ }^{2}$, que se enfoca en la formación de una delegación sindical y las estrategias de movilización de trabajadoras de aseo de una empresa en una universidad privada. Mi objetivo es explorar las condiciones de vida y trabajo de las trabajadoras subcontratadas de aseo y su proceso de sindicalización. Las preguntas que busco responder son: ¿Qué caracteriza a estos sujetos y sus condiciones laborales? Y ¿Cómo lograron organizarse?

Este artículo se organiza de la siguiente manera. En primer lugar, hago una revisión de la literatura relativa al trabajo subcontratado de aseo y las formas de organización de estas trabajadoras. En segundo lugar, repaso las perspectivas teóricas a partir de las cuales analizo los datos y respondo mis preguntas de investigación. Parto por la comprensión gramsciana de subalternidad y de las políticas subalternas. Luego reviso la intersección de dimensiones de género, clase y raza en este tipo de trabajo. Finalmente abordo el neoliberalismo, con un foco en la configuración de un estado neoliberal chileno y su impacto en el mercado del trabajo, particularmente en la externalización de servicios y en los ataques al sindicalismo. En tercer lugar, doy cuenta de la metodología y abordo cuestiones de locación y representación; describo el trabajo de campo que informó esta investigación y explico los procesos usados para recolectar, manejar y analizar mis datos. En cuarto lugar, en la sección de discusión muestro los resultados del análisis y los temas que emergen de las entrevistas. Finalmente, en las conclusiones resumo los principales argumentos y resultados de esta investigación, presento las contribuciones y limitaciones del artículo y elaboro posibles proyecciones.

\section{Servicios subcontratados de aseo}

El trabajo de aseo subcontratado ha sido abordado desde diferentes perspectivas teóricas. Una de ellas es la teoría del trabajo sucio (Dirty WorkTheory, en inglés), que plantea que las sociedades relegan las labores relacionadas con la suciedad a ciertas personas y luego se olvidan de ellas, excluyendo, marginalizando e invisibilizando a quienes se encargan de ella (Van Vlijmen, 2019). En su estudio sobre cómo trabajadoras de aseo en los Países Bajos lidian con el estigma del trabajo sucio, Van Vlijmen (2019) plantea que la valoración social de estas labores afecta su moralidad en tanto sirve de excusa para excluirlas y marginarlas y las hace sentir subhumanas. Soni- Sinha \& Yates (2013) realizaron un estudio con trabajadoras de aseo en Toronto y llegaron a conclusiones similares: la invisibilidad del trabajo sucio, junto con dimensiones de género y raciales, afectan sus condiciones laborales, porque sirve de justificación para tratos abusivos y bajos salarios.

Otra perspectiva desde la que se ha abordado el trabajo de aseo subcontratado es una aproximación interseccional que pone el foco en sus dimensiones de género y raza. Passos \& Nogueira (2018) lo analizan desde el contexto brasilero y plantean que, dado que reproduce la división sexual y racial del trabajo, recluta principalmente a mujeres afrodescendientes. El estudio de Soni- Sinha \& Yates (2013) mencionado anteriormente también aborda esta dimensión y plantea que en este tipo de labores existe una división

2 Este artículo se enmarca en la tesis realizada para optar al grado de Magister en Ciencias del Departamento de Género de la London School of Economics and Political Science el año 2019. 
de género que se intersecta con la dimensión racial de un trabajo realizado en su mayoría por sujetos migrantes y/o racializados. Las autoras dan cuenta de las formas en que jefes y administradores utilizan esto para controlar a las trabajadoras de aseo, lo que tiene un impacto tanto en sus identidades como en la percepción y valoración que otros hacen de su trabajo.

En Chile, la escasa investigación dedicada al trabajo de aseo subcontratado dentro de universidades está compuesta mayoritariamente por reportes enfocados en la descripción de sus condiciones laborales. Estos se enfocan en las razones que llevaron a universidades a externalizar sus servicios, la evaluación que hacen de este trabajo sus diferentes miembros, la caracterización sociodemográfica, laboral y contractual de las trabajadoras, los riesgos psicosociales que enfrentan y sus tasas de sindicalización. Estos muestran que estos servicios son realizados en su mayoría por mujeres pobres y que su sindicalización y organización son difíciles dadas las condiciones precarias de su trabajo y un miedo generalizado a estos procesos (Boccardo et al., 2015; Boccardo et al., 2016; Fundación Sol, 2014).

\section{La organización de trabajadoras de aseo subcontratadas}

No obstante, la literatura también muestra diferentes formas de organización y sindicalización que trabajadoras de aseo han llevado a cabo en diferentes contextos. Este ha sido el foco de estudios en el marco de las relaciones industriales que han estudiado las dificultades e imposibilidades de organización de estas trabajadoras debido a la estructura del subcontrato, a lógicas neoliberales y a sus condiciones de trabajo. Aguiar (2004) se enfoca en las políticas neoliberales contra el sindicalismo en Canadá que provocaron la marginalización, abuso, explotación e inseguridad de las trabajadoras de aseo. El autor también da cuenta de las formas en que estas trabajadoras enfrentaron la situación, ya sea reforzando sus antiguas formas de organización o reformulándolas en miras de formar alianzas con otras comunidades. Esto último la academia lo ha denominado sindicalismo comunitario, a saber, una forma de organización que, por sus alianzas con otros sectores del lugar de trabajo o de la sociedad, se acerca más a los movimientos sociales que a los sindicatos (Alberti, 2016; Herod \& Aguiar, 2006b; Hearn \& Bergos, 2011; Wills, 2008).

En esta línea, Alberti (2016) se enfoca en las movilizaciones de trabajadoras subcontratadas de aseo (en su mayoría migrantes) de la University of London. La autora plantea que el éxito de estas movilizaciones se debe al uso de estrategias propias de los movimientos sociales por fuera de los sindicatos tradicionales. No obstante, enfatiza en la importancia de estos últimos para legitimar y fomentar el poder de estas trabajadoras. En un contexto similar, Hearn \& Bergos (2011) se enfocaron en una movilización de trabajadores subcontratadas de aseo realizada en 2008 en SOAS University en que participaron trabajadoras, sindicatos, organizaciones comunitarias y estudiantes. Los autores plantean que las estrategias de sindicalismo comunitario fueron centrales para el éxito de esta campaña. No obstante, destacan la importancia de los sindicatos tradicionales, puesto que la sindicalización de las trabajadoras de aseo mostró ser 
fundamental para poder protegerlas legalmente de los contrataques perpetrados por las empresas, especialmente hacia trabajadoras migrantes.

No hay estudios que aborden procesos de movilización, organización o sindicalización de trabajadoras subcontratadas de aseo en Chile. No obstante, los trabajadores subcontratados de diferentes rubros (minería, forestales, pesca y puertos) se han movilizado y organizado desde los años 80 (Echeverría, 2010) bajo formas novedosas que han llevado a académicos y movimientos sociales a hablar de un nuevo sindicalismo (Echeverría, 2010; 2013; Abarzúa, 2008; Calderón, 2008; Ruiz \& Boccardo, 2014). Este emerge como una respuesta a las formas neoliberales de precarización del trabajo y difiere del sindicalismo tradicional en que se basa en la construcción de una identidad social o colectiva no fundamentada en la clase, sino en las injusticias y en la precarización inherentes al sistema de subcontratación (Abarzúa, 2008; Calderón, 2008; Echeverría 2010; 2013). Según Abarzúa (2008), las movilizaciones en este marco son intensas, pero corren el riesgo de terminar una vez que alcanzan sus metas. Además, los trabajadores organizados bajo estas lógicas no tienen una estructura organizacional rígida, sino que actúan a nivel interempresa o nacional, y generalmente el contenido de sus campañas es cualitativo. En esta línea, sus acciones colectivas son más radicales, buscan apoyo en otros actores sociales y tienen relaciones tensas con los sindicatos de empresa con los que conviven (Abarzúa, 2008; Caldeón, 2008). Este nuevo sindicalismo se ha expandido a otras áreas como la agricultura (Valdés, Godoy \& Mendoza, 2017) o el retail y ha modificado el panorama laboral chileno (Echeverría 2010; 2013).

\section{Marco teórico}

I. Subalternidad y políticas subalternas

Gramsci (1996) acuñó el término "subalterno" durante sus años en la cárcel para referirse a la subordinación y marginalización social de clase generada por relaciones políticas, sociales, culturales y económicas de poder. Este concepto le permitió expandir el concepto marxista de proletariado y clase trabajadora (Liguori, 2015). Para Gramsci (1996) la subalternidad es el equivalente sociopolítico de la alienación y se opone a la hegemonía. El término refiere a la desposesión relativa de la cualidad subjetiva a través de la subordinación sociopolítica (Gramsci, 1996). Los subalternos asimilan su subordinación a través de la internalización de los valores hegemónicos y las ideologías impuestas por las clases sociales dominantes (Green, 2002; Modonesi, 2012). Según Gramsci (1996) en el marco del estado integral, la hegemonía se genera a través de consensos que son, a su vez, protegidos mediante coerción (y viceversa) para resguardar las posiciones políticas y económicas de las clases dominantes.

El autor entendía al subalterno como contextual e históricamente específico e identificó como tales a diferentes grupos: personas esclavizadas, campesinos, mujeres, sujetos racializados, trabajadores industriales, entre otros (Green, 2002). Estos grupos varían según sus niveles de organización política y se caracterizan por su heterogeneidad, disgregación, por la espontaneidad de sus acciones políticas y por una tendencia débil hacia la unidad (Gramsci, 1996; Modonesi, 2012; Green, 2002). Sin 
embargo, la subalternidad es también el punto inicial de conflictos y procesos de emancipación que se componen de seis fases que dan cuenta del desarrollo desde una oposición primitiva de subordinación hacia la autonomía, en tanto grupo social dominante (Gramsci, 1996; Green, 2002). Hasta que no alcanzan esa última fase y desarrollan un nuevo sentido común que transforme la sociedad, seguirán siendo sometidos a las iniciativas de la clase dominante, incluso cuando intenten rebelarse (Gramsci, 1996).

Los subalternos han desafiado la subalternidad desde abajo en diversos momentos históricos (Nilsen \& Roy, 2015). Acciari (2018) presta atención a trabajadoras domésticas en Brasil y a sus procesos de movilización y sindicalización. La autora las caracteriza como como sujetas subalternas por la intersección de factores de género, raza y clase en un contexto poscolonial, pero sugiere que, paradójicamente, su subalternidad le da forma a su identidad colectiva y a sus repertorios de acción. Bayat (2000) se enfoca en las consecuencias económicas de la globalización en ciudades del tercer mundo y estudia las movilizaciones de personas pobres en zonas urbanas, a quienes caracteriza como subalternos. El autor plantea que estos grupos realizan lo que él llama una "invasión silenciosa", a saber, una acción directa no colectiva pero prolongada, mediante la cual los individuos y familias marginalizadas adquieren servicios básicos discreta e ilegalmente.

II. Interseccionalidad

Crenshaw (1989) acuñó el concepto de interseccionalidad para referirse a una herramienta, un lente analítico crítico que permite abordar las formas particulares de subordinación que han experimentado las mujeres afrodescendientes, evitando el ejercicio de sumar estructuras opresivas. En este sentido, la interseccionalidad ayuda a evitar enfocarse en una sola estructura opresiva a la vez, pasando por alto los efectos que estas tienen entre sí (Spelman, 1990). Además, permite capturar las partes comunes de las experiencias de mujeres afrodescendientes y sugiere que estas pueden dar luces sobre las experiencias de otros grupos marginalizados (Cooper, 2016). Así, en tanto metodología, considera las diferentes formas en que modos de poder se intersectan y ofrece herramientas para desarmarlas (Cooper, 2016).

En este artículo, la interseccionalidad permite analizar las estructuras de poder intersectantes que están en juego en el trabajo de aseo subcontratado. Este es un tipo de trabajo de servicio institucional, es decir, trabajo reproductivo en entornos institucionales (Duffy, 2007) marcado por dimensiones de género, raza y clase (Nakano Glenn, 1992). En tanto parte de la reproducción social, es decir, de las diversas actividades y relaciones que sostienen la vida de las personas a diario e intergeneracionalmente (Nakano Glenn, 1992; Duffy, 2007, Fraser, 2016) y por reproducir la colonialidad del trabajo que restringe ciertos cuerpos a trabajos particulares (Lugones, 2007), estos trabajos están altamente devaluados, son escasamente consideras trabajo "real" y son realizados en su mayoría por mujeres racializadas o pertenecientes a minorías étnicas (Nakano Glenn, 1992).

En su estudio sobre las experiencias de trabajo de mujeres migrantes afrocolombianas en el norte de Chile, Segovia, Ramírez-Aguilar \& Zapata-Sepúlveda (2018) ilustran que estas se dedican mayoritariamente al servicio doméstico, al aseo industrial y a la cocina. En esta línea, el reporte de la Fundación Sol sobre las condiciones 
del trabajo subcontratado en una universidad estatal en Santiago muestra que el $11 \%$ de la fuerza de trabajo era migrante. A esto se suma que la mayor parte de otros trabajos institucionales de servicio, como el trabajo doméstico, sea realizado por mujeres mapuche o migrantes (Antileo, 2015; Boccardo et al., 2018; CASEN, 2016) como consecuencia de la colonialidad del trabajo que relega a la población indígena a "trabajos para indios" (Antileo, 2015) y a la población migrante a trabajos precarios (Castillo, Esnaola, López, Ratto, \& Seguel, 2015). Es importante tener en cuenta estos factores al abordar el trabajo de aseo subcontratado, especialmente con el aumento de la migración en el país (Departamento de Extranjería y Migración, 2018). Estas características se tornan aún más complejas si se toma en consideración el neoliberalismo y la subcontratación.

III. Neoliberalismo y subcontratación

Siguiendo a Harvey (2007a; 2007b) —quien plantea que el neoliberalismo es una teoría de prácticas político-económicas que proponen como la mejor forma de alcanzar el bienestar de los humanos la liberación de habilidades y libertades empresariales individuales, en un marco institucional caracterizado por derechos de propiedad privada sólidos, libre mercado y libre comercio—, y Brown (2015) — para quien es una racionalidad de gobierno que transforma cada esfera de la actividad humana en términos económicos-en este artículo voy a entender el neoliberalismo como un conjunto hegemónico de políticas económicas que produce y es producido por una gubernamentalidad neoliberal.

Las políticas neoliberales fueron impuestas (a través de la fuerza, el consenso o ambas) para restaurar el poder de las élites económicas (Harvey, 2007; Brown, 2015; Ruiz \& Boccardo, 2014) a través de lo que Harvey (2007a, 2007b) denomina acumulación por desposesión. En el caso chileno, el neoliberalismo fue impuesto durante la dictadura cívico-militar a través de represión y diversas reformas (Harvey, 2007; Brown, 2015; Ruiz \& Boccardo, 2014). Postdictadura, los gobiernos democráticos modificaron de algunas formas estas políticas neoliberales a la vez que profundizaron otras (Ruiz \& Boccardo, 2014).

Paralelo a estas políticas neoliberales, y como una forma de asegurarlas, el neoliberalismo, en tanto racionalidad de gobierno, se ha ido expandiendo en el país desde la dictadura, imponiéndose silenciosamente y volviéndose parte del sentido común que apela a ideas políticas como la dignidad humana y la libertad individual (Harvey, 2007). Así, ha ido extendiendo formulaciones específicas de valores y prácticas económicas a cada dimensión de la vida y las actividades humanas, configurando a los seres humanos como homo oeconomicus, esto es, como agentes económicos exhaustivos e implacables marcados por el emprendimiento, cuyo proyecto es invertir en sí mismos para potenciar su valor en cada esfera de su existencia (Brown, 2015). El neoliberalismo reemplaza el intercambio por la competencia, lo que, combinado con políticas que precarizan a los sujetos, fomenta el individualismo y previene la participación política (Brown, 2015). De este modo, el neoliberalismo se ha vuelto hegemónico en tanto modo discursivo (Harvey, 2007a). Así, a través de subordinación y desposesión de la calidad subjetiva (Modonesi, 
2012) el neoliberalismo produce sujetos subalternos. Uno de los mecanismos que utiliza para ello es el subcontrato.

Las políticas neoliberales reestructuraron el mercado del trabajo mediante la flexibilización (Harvey, 2007), facilitando el subcontrato (Herod \& Aguiar, 2006; de la Garza, 2012). En Chile, contrario al resto de Latinoamérica (Iranzo \& Richter, 2012) y otros países como Canadá (Herod \& Aguiar, 2006a), el subcontrato no emerge como respuesta a los cambios en la estructura de producción. Por el contrario, fue impuesto durante la dictadura a través de reformas que buscaban desregular el mercado del trabajo (Iranzo \& Richter, 2012; Harvey, 2007a). Así, el subcontrato fue implementado en el marco de la neoliberalización del Estado como una forma de debilitar el sindicalismo y socavar la democracia. Esto fue paralelo a otras reformas relativas a la sindicalización y negociación colectiva, que previnieron la posibilidad de negociar por rama y limitaron la negociación al marco de las empresas (Echeverría 2010; 2013).

IV. En el caso chileno, el trabajo subcontratado es aquel

"realizado en virtud de un contrato de trabajo por un trabajador para un empleador . . . cuando éste, en razón de un acuerdo contractual, se encarga de ejecutar obras o servicios, por su cuenta y riesgo y con trabajadores bajo su dependencia, para una tercera persona natural o jurídica dueña de la obra, empresa o faena" (Artículo 183-A del Código del Trabajo).

El subcontrato tiene múltiples consecuencias negativas que se relacionan entre sí, tales como deteriorar las condiciones laborales, generar desigualdades entre los trabajadores de la empresa principal y los subcontratados, debilitar relaciones laborales individuales y colectivas, y afectar la sindicalización y la negociación colectiva (Echeverría, 2010; 2013; Iranzo \& Richter, 2012; de la Garza, 2012). Lo último se torna aún peor considerando que el Estado neoliberal chileno, junto con otras reformas, removió la seguridad social y delegó la responsabilidad de la reproducción social a los individuos (Ruiz \& Boccardo, 2014; Harvey, 2007a).

En Chile, como en otros lugares del mundo en los que la desigualdad devastadora del neoliberalismo es la regla, estas situaciones afectan la supervivencia de los trabajadores (Ruiz \& Boccardo, 2014). Su trabajo los desposee y apenas les otorga los medios para sobrevivir. En este sentido, el subcontrato se vuelve una trampa para los "perdedores del neoliberalismo" (Brown, 2015): no pueden escapar de él porque no tienen los medios para sobrevivir. Más aún, no pueden alcanzar movilidad social o mejores sueldos porque no tienen entrenamiento y su trabajo es devaluado (Echeverría, 2010). De este modo, el subcontrato se torna un mecanismo neoliberal de producción de sujetos subalternos. 


\section{Metodología}

I. Métodos cualitativos y epistemología feminista

Este artículo explora los elementos laborales y de vida que configuran a trabajadoras de aseo subcontratadas como sujetas subalternas y sus posibilidades de organización. Para ello, narrar dicho proceso "desde abajo" y relevando sus voces se torna crucial. En un esfuerzo por ser lo más fiel posible a sus voces y a sus propias percepciones, esta investigación opta por un método cualitativo que permite obtener datos de las perspectivas, emociones, experiencias, significados y otros elementos subjetivos de las trabajadoras. Además, permite entender que quien investiga y los sujetos de su investigación se influencian mutuamente y no pueden ser del todo separados. En este sentido, quien investiga hace explícita su postura, reconoce sus valores y creencias y los hace parte del estudio (Hernández, Fernández, \& Baptista, 2010). Por estas razones, se trata de una metodología acorde con epistemologías y metodologías feministas que le dan importancia a la subjetividad y plantean la imposibilidad de la objetividad o la universalidad (Madhok\& Evans, 2014).

Una de las contribuciones más significativas de la epistemología feminista es el concepto de locación. Este hace referencia a que hay elementos de la vida, lugar y posición de quien investiga que marcan tanto su conocimiento como su producción. Mohanty (1991) plantea que la locación fomenta y permite modos específicos de leer y de conocer lo dominante. En esa línea, Rich (1986) propone que influye en cómo una persona crea y trata de crear. Siguiendo estas ideas y en un intento de ser reflexiva, a continuación, abordo algunos elementos claves de mi propia locación y cómo afectan mi investigación.

Mi relación con las trabajadoras se vio impactada desde un comienzo por el hecho de ser, en su momento, una estudiante de clase alta, pero también por ser de izquierda y feminista. Durante el pregrado, mi advocación por un feminismo de clase, entonces incipiente, me llevó a participar en un proyecto estudiantil que buscaba, a través de la educación popular, aportar en la escolarización de las trabajadoras subcontratadas de aseo en miras de un eventual proceso de internalización. A través de dicho proyecto y del trabajo colectivo, junto con compañeros, comenzamos a ganarnos la confianza de muchas de ellas. Después de dos años de trabajo y convivencia, la relación que habíamos construido era de una amistad y complicidad profunda. Estos elementos impactaron mi investigación de formas que iré profundizando en el resto de esta sección.

II. Herramientas, muestra y análisis

Este estudio se basa en cuatro entrevistas semiestructuradas, la observación participante de diferentes instancias (reuniones, asambleas de sindicato y encuentros con trabajadoras) y la revisión de documentos sindicales facilitados por la antigua presidenta del sindicato durante un trabajo de campo de dos semanas realizado en junio de 2019. Las entrevistas abordaron temas relativos a su vida doméstica y laboral, la percepción de la división sexual del trabajo, la formación de la delegación sindical, los hitos de su organización y la huelga que realizaron el 2018. Además, dos entrevistas realizadas a 
dirigentas abordaron temas relativos a representatividad y liderazgo. Las entrevistas fueron grabadas previo consentimiento, anonimizadas y transcritas junto con las notas de campo. Luego, fueron codificadas en un software de análisis cualitativo (Atlas.ti) y analizadas a través de un método mixto de teoría fundamentaday análisis narrativo (Hernández et al., 2010).

La relación cercana con las trabajadoras facilitó mi inserción en el campo, sobre todo en su disposición a participar de mi investigación, pero también me enfrentó a algunas complicaciones inesperadas, como tener dificultades para distanciarme emocionalmente de las trabajadoras en tanto sujetos de estudio, y de mis experiencias y conocimientos previos de todo el proceso, para escuchar lo que ellas tenían para decir y no lo que yo quería oír. No obstante, una de las mayores dificultades respondió a un elemento particular de mi locación a saber, mi blancura, en tanto mis primeras aproximaciones a esta investigación no tomaron en cuenta la raza como una dimensión relevante a considerar. Esto, sobre todo, porque en el sindicato había una sola mujer mapuche, quien nunca mencionó haber sido discriminada en el trabajo por ello, y ninguna otra persona racializada. No obstante, durante la revisión de literatura y en conversaciones con compañeras feministas, comencé a explorar la colonialiad del trabajo en el contexto chileno. Entonces entendí que mi suposición estaba contribuyendo a la invisibilización tanto de grupos sociales como de relaciones de poder. Lamentablemente, mi investigación no pudo profundizar en esta dimensión por falta de datos.

\section{Representación y autenticidad}

Otro aspecto donde latían las relaciones de poder en mi investigación fue respecto a temas de representación y autenticidad. La representación contempla la descripción y entrega de información respecto aquellas personas sobre las que se habla, pero también implica la producción de una imagen de ellos, sus experiencias y realidades (Spivak, 1988; Mohanty, 1991). La representación, entonces, en tanto forma de poder, supone desafíos y problemas relativos a autenticidad, responsabilidad, ética y poder: ¿Quién puede representar a quién? ¿Dónde? ¿De qué formas? ¿Con qué efectos/consecuencias para aquellos representados? Según Alcoff (1995), este tipo de problemas ha llevado a algunas académicas a argumentar a favor de abstenerse de representar. No obstante, dicha abstención tampoco está libre de problemas, ya que reproduce privilegios (solo algunos tienen la opción de hacerlo) (Alcoff, 1995), permite que investigadores menos críticos continúen produciendo y reproduciendo representaciones problemáticas (Pedwell, 2002) y finalmente solo sirve para expiar en cierta medida la "culpa privilegiada" (Mullassery, 2002).

Las trabajadoras subcontratadas de aseo pueden y se han representado a sí mismas en múltiples contextos. No obstante, no lo han podido hacer en el contexto particular de la academia debido a relaciones de poder que reproducen jerarquías que, como a otros sujetos, las excluyen de estas esferas (Kilomba, 2013). La intersección de estructuras opresivas y las condiciones laborales que las configuran como subalternas las tornan mudas en este contexto particular. No obstante, dada la importancia de su historia y mi responsabilidad política de denunciar y hacer frente a la opresión (Alcoff, 1995) y de 
cuestionar las marginalidades epistémicas (Madhok \& Evans, 2014) me atrevo a representarlas, con su permiso ${ }^{3}$, usando sus voces y mis propias interpretaciones (Alcoff, 1995). No obstante, esto supone otro problema más: la autenticidad. No me es posible reproducir de manera completamente fiel sus voces y no pretendo hacerlo tampoco. $\mathrm{Mi}$ locación siempre tendrá un efecto sobre ellas (Alcoff, 1995; Spivak, 1988). No obstante, siguiendo a Spivak (1988) y a Acciari (2018), en este texto me propongo trabajar con las subalternas para representar (imperfectamente) algunas de sus luchas (Acciari, 2018) hablando con ellas (Spivak, 1988), escuchándolas y considerando sus experiencias como fuentes válidas de conocimiento (Acciari, 2018).

\section{Resultados}

I. Sujetas subalternas bajo condiciones neoliberales precarias de trabajo

El subcontrato produce condiciones precarias de trabajo, particularmente cuando lo que se externaliza son trabajos poco especializados, permanentes y adyacentes al rubro principal de la empresa (Echeverría, 2010). Este es el caso de la universidad que compete a este estudio, que decidió externalizar ciertos servicios por los beneficios de la flexibilización (Boccardo et al., 2016) luego de la economización neoliberal (Brown, 2015) que transformó a las universidades en empresas (Downey \& Portiño, 2017).

Las condiciones laborales de las trabajadoras de aseo al interior de esta universidad son precarias: no siempre cuentan con todos los implementos necesarios para realizar su trabajo, deben cambiarse de ropa y comer en espacios con una infraestructura inapropiada, sus sueldos y los beneficios a los que pueden acceder son mucho más bajos que los de los trabajadores de planta, cuestión que resienten profundamente.

Eh, lo que más odiaba sí yo era ir a botar la basura a los container, porque teníai que reciclar las bolsas... de los tarros, . . . entonces dar vuelta todas esas bolsas, . . yo sí hacía llegaba a mi casa y me bañaba al tiro antes de. Yo en el trabajo no me bañaba porque no encontraba que los baños eran aptos para los trabajadores tampoco, yo llegaba a mi baño, a la casa con asco así y llegaba al tiro a bañarme, porque sentía que iba con la mugre, con, yo llegaba con el olor impregnado a mi casa, o sea me sentía el olor a cuando day vuelta la basura en los tachos." (Constanza, 2019)

Estos elementos contribuyen a su marginalización de la universidad, lo que se ve reforzado a su vez por malos tratos por parte de estudiantes, profesores y otros

\footnotetext{
${ }^{3}$ Durante mis conversaciones con las trabajadoras, al explicarles mi investigación, les conté que había algunas corrientes académicas que plantean que, debido a sus condiciones materiales, las trabajadoras subcontratadas no se pueden organizar y menos aún formar un sindicato. Muchas de ellas se impactaron con esto y plantearon que es difícil, pero se puede y es importante que la gente lo sepa.
} 
miembros. Las relaciones con la empresa no son mejores. Las trabajadoras eran constantemente acosadas, amenazadas, insultadas, explotadas y maltratadas. ${ }^{4}$

La supervisora . . nos trataba súper mal. Yo me acuerdo que no alcancé a estar un mes y me tuve que ir con licencia, no estaba acostumbrada al maltrato y fue, fue terrible para mí, po. Entonces después era, yo veía a la supervisora y era... era tiritar de verla" (Mónica, 2019)

Estos abusos psicológicos también tenían consecuencias físicas, como cuando una supervisora golpeó a una trabajadora. A esto se suma que tanto supervisoras como jefes les prohibían hablar con otros miembros de la universidad, lo que contribuía aún más a la marginalización propia del subcontrato. Cuando las trabajadoras se atrevían a reportar o denunciar estas situaciones, eran amenazadas, rotadas de instalación o despedidas. Estos abusos, combinados con la hegemonía neoliberal prevalente en el país que las torna homo oeconomicus caracterizados por el individualismo y la competencia (Brown, 2015), impacta en las relaciones entre trabajadoras. Con un Estado neoliberal que las hace responsables de su propio bienestar (Harvey, 2007a; Brown, 2015) en un contexto en que son absolutamente dispensables (Brown, 2015) y penden de contratos inseguros, las trabajadoras tienden a enfocarse en sí mismas, lo que deja poco espacio para la cooperación y la solidaridad (Notas de campo, 2019).

Además, dado que no reciben suficiente capacitación u otras formas de aumentar su valor de mercado, lo intentan a través de imprimir su sello en su trabajo y hacerlo de la mejor manera posible. En este marco, las otras trabajadoras no solo aparecen como competencia, sino también como obstáculos para alcanzar este objetivo. Las trabajadoras acusan constantemente que sus compañeras aumentan su carga de trabajo por "flojas" o por "hacer mal la pega". Además, compiten por las horas extra (Boccardo et al., 2016). Las supervisoras y jefes aprovechan estos conflictos para profundizar aún más sus malas relaciones, inventando rumores o generando peleas. Cuando estas estrategias en la lógica de "divide e impera" no funcionan y las trabajadoras logran generar vínculos, la empresa recurre a rotaciones o despidos para separarlas. Estas relaciones de trabajo autoritarias dificultan aún más su escasa tendencia a la unión debido a los obstáculos legales que el subcontrato impuso a la sindicalización (Echeverría, 2010; Ruiz \& Boccardo, 2014). Una vez sindicalizadas, estos mismos elementos suponen una amenaza constante para la estabilidad del sindicato (Notas de campo, 2019). Así, las características del trabajo subcontratado sumada a una gubernamentalidad neoliberal, marginalizan y oprimen a las trabajadoras y las vuelven sujetos subalternos en el sentido gramsciano.

II. La intersección de género, clase y raza

Los elementos anteriores no son los únicos que contribuyen a su subalternidad. Las trabajadoras de aseo de esta universidad, en su mayoría mujeres cuyos sueldos deben alimentaren promedio a tres personas, están adeudadas y sus niveles

\footnotetext{
${ }^{4}$ Construyo esta oración en pretérito porque muchas de estas situaciones particularmente graves cesaron gracias a la acción de trabajadoras, el sindicato y el apoyo de estudiantes.
} 
educacionales son bajos (Boccardo, et al., 2016). Estos factores de clase intersectan con dimensiones de género y raza que empeoran su marginalización y opresión.

Según Nakano Glenn (1992), en Estados Unidos, el trabajo institucional de servicios recluta mayoritariamente a mujeres racializadas o de minorías étnicas en función de una división de género y de raza. Esta situación se repite en otros países donde las mujeres migrantes y afrodescendientes dominan la industria del aseo (Alberti, 2016; Passos \& Nogueira, 2018; Soni - Sinha \& Yates, 2013). En el caso de esta universidad, hasta el 2016, el 7\% de las trabajadoras era extranjera (Boccardo et al., 2016) ${ }^{5}$. No obstante, el informe no especifica sus orígenes ni el número de trabajadoras de ascendencia indígena. Una de las entrevistadas para este estudio es mapuche, pero, a diferencia de lo que plantea la literatura, no estaba relegada a "trabajos para indios" (Antileo, 2015) y, según ella, nunca experimentó algún tipo de discriminación por ello (Constanza, 2019). No obstante, sí ha habido casos de discriminación y racismo que han empeorado las ya deplorables condiciones laborales de las trabajadoras de aseo al interior de esta universidad. Tal fue el caso de una trabajadora migrante que fue despedida sin compensaciones y fue amenazada con ser deportada si ejercía sus derechos legales (Defensoría Popular de las y los Trabajadores, 2018).

Según Boccardo et al. (2016) la mayor parte de las trabajadoras de la universidad $(90,5 \%)$ son mujeres, lo que reproduce la feminización del trabajo reproductivo (Fraser, 2016; Nakano Glenn, 1992). Además, sigue la lógica de relegarlas a trabajos precarios y mal pagados (Riquelme \& Abarca, 2015). Esto se ve empeorado por el hecho de que la mayor parte de estas mujeres son las únicas proveedoras de sus hogares y deben realizar el trabajo reproductivo en ellos también (Boccardo et al. 2016; Constanza, 2019). Como ilustran Boccardo et al. (2016), entre los riesgos sociales a los que están expuestas, la doble presencia, es decir, la exposición a una doble jornada (industrial y doméstica) presenta los más altos índices de trabajadoras en riesgo psicosocial. Además, muchas de ellas tienen dificultades para diferenciar los problemas de la casa respecto de los del trabajo, lo que no solo tiene un impacto en su salud, sino también en las relaciones entre compañeras (Vanesa, 2019; Constanza, 2019) y, por ende, en sus posibilidades de organización.

Hay otra dimensión de este trabajo que refuerza aún más la subalternidad de estas trabajadoras, a saber, su valoración. Según las entrevistadas, ni la empresa ni la universidad valoran el trabajo que realizan. Esto no solo se debe a que el suyo es un trabajo reproductivo que en general está devaluado socialmente (Nakano Glenn, 1992), sino, como ellas mismas plantean, a que trabajan con suciedad.

Porque te toman como si fuerai lo último de abajo, que fuerai lo peor de abajo, del trabajo que tú hacís (Mónica, 2019).

Otra entrevistada también abordó estas dimensiones:

\footnotetext{
${ }^{5}$ Este informe realizado por la Fundación Nodo XXI describe las condiciones laborales y de vida de trabajadoras y trabajadores de 16 empresas al interior de la universidad. No obstante, mi análisis se limita a las trabajadoras contratadas por una de ellas.
} 
C: Yo no sé de repente veo en... como que las señoras del aseo no más, las tías del

E: ¿Qué te nada pasa más. con del del aseo?

C: Mmm aseo... como que... bueno es una tontera no más, como que hacer aseo como que es lo último ya ¿cachai? Es lo último. (Celia, 2019)

No es solo su trabajo es devaluado por ser considerado sucio (Van Vlijmen, 2019), sino que ellas, como sujetos, son devaluadas también. Esto contribuye a marginalizarlas aún más y afecta cómo las personas interactúan con ellas, como en el caso de las trabajadoras analizadas por Vlijmen (2019) y Soni-Sinha \& Yates (2013).

Hasta un cierto punto estas trabajadoras aceptan su subalternidad, en tanto se ha convertido en parte de su sentido común en un contexto nacional donde el neoliberalismo es hegemónico. Como he mostrado hasta este punto, tanto a través de coerción como de consentimiento, son disciplinadas para aceptar condiciones laborales miserables. La actitud que predomina es "mientras me paguen yo estoy bien" (Celia, 2019). No obstante, como plantea Gramsci (1996), y como muestra Acciari (2018), es precisamente dicha subalternidad la que sirve de puntapié inicial para sus posibilidades de emancipación. La historia de organización y sindicalización de estas trabajadoras es ejemplo de aquello.

\section{¿Puede la sujeta subalterna organizarse?}

Como he mostrado a lo largo de este artículo, el subcontrato no solo deteriora las condiciones laborales, sino también los derechos laborales colectivos a través de la desintegración de la comunidad laboral. En Chile, las políticas neoliberales contra el sindicalismo empeoraron esta situación. En el caso de las trabajadoras de aseo, estas posibilidades se dificultan aún más debido a su subalternidad. No obstante, las trabajadoras de esta investigación se organizaron, formaron un sindicato y realizaron una huelga que tuvo impactos materiales y cualitativos positivos, como mencionaron todas las entrevistadas.

\section{Movilizar la subalternidad}

En 2017, Vanesa, una trabajadora subcontratada de aseo cansada de los abusos de su supervisora decidió tomar el control de la situación y "arriesgar[se] no más, po, dejar [su] cobardía y seguir adelante" (Vanesa, 2019). Unió fuerzas con Mónica, quien también había sufrido acosos por parte de esa supervisora, y juntas buscaron frenar los abusos, pero no sabían cómo y los intentos individuales previos habían fallado (Vanesa, 2019). Con ayuda de estudiantes, decidieron formar un nuevo sindicato ${ }^{6}$ que pudiera proveer los medios legales y la fuerza para demandar a la supervisora, a la empresa y a la universidad por abuso laboral. El desafío no era menor, sobre todo por el miedo y frustración que sentían. Formar un sindicato y demandar era muy riesgoso (las trabajadoras ya habían tenido la experiencia de despidos cuando se estaba formando el sindicato empresa). Además, la ley chilena dificultaba sus intentos de formar un sindicato,

\footnotetext{
${ }^{6}$ El año 2015 se formó el primer sindicato empresa dentro de una de las empresas de aseo de esta universidad. No obstante, según las trabajadoras, se trata de un sindicato "proempresa" cuyas dirigentas son en su mayoría supervisoras que se protegen entre sí.
} 
puesto que impone un mínimo de 25 personas para su formación (Echeverría, 2010) y para entonces las trabajadoras no contaban con tanta gente. Así, la opción más viable para ellas era unirse a un sindicato interempresa, que por anonimidad llamaré SINDICATO, y formar una delegación sindical, para lo cual solo necesitaban ocho trabajadores.

Según las trabajadoras, unirse a SINDICATO no fue fácil por miedo y desconfianza. Para convencer a otras trabajadoras, Vanesa, con el apoyo de estudiantes, abogados y miembros de SINDICATO, tuvieron que hacer un "trabajo de hormiga" (Vanesa, 2019) para explicarles sus derechos laborales y las estrategias que se podían desplegar para protegerlas (por ejemplo, a través de la garantía de indemnidad). En mayo de 2017, ocho trabajadoras superaron sus miedos y decidieron formar la delegación. En junio eligieron a Constanza como su delegada. Luego de múltiples denuncias a la inspección del trabajo, la incorporación de nuevas socias y un arduo trabajo de organización junto a estudiantes y dirigentes de SINDICATO, la delegación logró mejorar las condiciones materiales de trabajo y en enero de 2018 ganaron el juicio contra la empresa por acoso laboral. La supervisora abusiva estaba fuera. Este logro las llenó de fuerzas y esperanza y las motivó a iniciar una negociación colectiva con la empresa y posterior huelga en abril de 2018.

Fue emocionante que la hayan sacado, fue como un... como haber sacado... una como te dijera una mochila de una persona maltratadora. . . y al sacarla se empezó a arreglar todo po y eso fue emocionante para muchos, po, para mí, para muchas compañeras, que la vivieron mal, incluso para compañeras que supieron que se había ido, que se había logrado, ... fue emocionante ver que, todos los que nos miraron en menos ahora dijeron 'valía la pena'. Fue todo lento, pero... todo... valorable. (Vanesa, 2019).

Su sindicalización tuvo el carácter espontáneo de los grupos subalternos que no han alcanzado una conciencia de clase como tal (Gramsci, 1996). Estas trabajadoras no se unieron en torno a una identidad colectiva (ya sea de clase o de trabajadoras de aseo) - de hecho, tendían a medir su participación en el sindicato en términos económicos, reproduciendo la lógica de la participación democrática mediada por razones económicas e individuales (Brown, 2015)—, sino en función de una experiencia común de abuso. En este sentido, su subalternidad, como en el caso de las trabajadoras domésticas en Brasil (Acciari, 2018) o los trabajadores subcontratados del cobre (Abarzúa, 2008), es paradójica: las une y moviliza como grupo, a la vez que es lo que quieren rechazar y superar. Su motivación era personal, pero devino colectiva por una experiencia común. Así, un elemento que contribuía a su subalternidad fue la fuerza que motivó su organización. A diferencia de otros casos del nuevo sindicalismo chileno que comparten esta característica (ver Abarzúa, 2008; Calderón, 2008) la espontaneidad y fuerza de su lucha no disminuyó una vez que alcanzaron este objetivo, por el contrario, las motivó a ir por más: salarios, beneficios y respeto. Las trabajadoras alcanzaron algunas de estas metas mediante denuncias en la inspección del trabajo, protestas dentro y fuera de la universidad y una negociación colectiva y posterior huelga en la que participaron 23 socias. 


\section{Formando alianzas con otros actores}

Según las trabajadoras, las alianzas con los estudiantes fueron fundamentales. Las y los estudiantes adoptaron medidas para ayudar a protegerlas del acoso que sufrían, tales como establecer cuadrillas para acompañarlas durante el turno de noche (el más propenso a que se dieran abusos por la menor presencia de gente en los campus). Esto las hizo sentir seguras. Además, los estudiantes y las trabajadoras se trataban como iguales en un contexto en que esto no es la norma, teniendo un impacto fundamental en revertir la marginalización que experimentaban. Finalmente, recurrir a los estudiantes tuvo un sentido más práctico durante marchas, protestas y la huelga, ya que aportaban con la masividad que necesitaban para exigir a la universidad y la empresa una mejora en sus condiciones laborales y muchas veces sirvieron de mediadores entre estos actores.

EI SINDICATO también fue una alianza fundamental. Según las trabajadoras, su relevancia, poder, recursos, acceso a apoyo legal y el uso de mecanismos de acción directa fueron cruciales para sus logros como delegación. Con su apoyo, las trabajadoras realizaron una huelga, realizaron corta calles, protestaron en la universidad y fuera de la empresa, y llenaron esta última de basura. Esto último fue un desahogo gigante para ellas (Constanza, 2019) y simbólicamente poderoso: la basura con la que llenaron la empresa representaba el trato que recibían y sirvió como una forma de revertir su subordinación. Ellas ya no estaban ahí para limpiarla sumisamente, sino para usarla rebeldemente contra la empresa.

Otro factor clave fueron las características de la delgada sindical. La preocupación constante de Constanza por sus compañeras y su devoción "24/7" como decía ella, por las labores sindicales, mantuvo al grupo unido y al sindicato funcionando. Un elemento esencial de su liderazgo era el hecho de ser "psicóloga, psiquiatra y mamá" (Constanza, 2019) de sus compañeras. Estos son estereotipos típicamente atribuidos a líderes mujeres (Cid, 2012; Riquelme \& Abarca, 2015), pero que pueden ser usados estratégicamente (Cid, 2012). De hecho, Constanza usaba su rol materno para alcanzar ciertos objetivos. Ella misma destaca su habilidad para escuchar "calmadamente" al hablar con la gente como algo que la ayudó en sus relaciones con autoridades y socias. No obstante, estos fueron roles que se le impusieron también para hacerse cargo de problemas que no tenían relación con el sindicato (ej. contestar llamadas a altas horas de la madrugada para escuchar problemas familiares). Esta demanda, sumado a ser mamá y al trabajo reproductivo que realizaba en su casa, "pelar papas y revisar correos, lavar loza y contestar llamadas" (Constanza, 2019), estar a disposición del sindicato todo el tiempo y sentir que su trabajo no era valorado por las socias, terminaron por afectarla psicológicamente y la forzaron a renunciar. Esta situación encarna otra paradoja: mientras Constanza era fundamental para la organización del sindicato y para superar ciertas formas de opresión, su liderazgo y las demandas de las trabajadoras reforzaron estructuras opresivas, sobre todo de género, en su vida.

Todos estos actores contribuyeron al empoderamiento de las trabajadoras de aseo, lo que a su vez las ayudó a superar el miedo y afiató el sindicato. Cada éxito y la apreciación que recibían de otras personas por ello, las ayudó a ganar un respeto que 
ellas sentían merecían y que valoran como el mayor logro de su organización. Este empoderamiento también se relaciona con su autovaloración como trabajadoras. Ellas se resisten a la devaluación de su trabajo y al estigma del trabajo sucio al considerarlo fundamental para el funcionamiento de la universidad y de la empresa. Las trabajadoras sostienen que el suyo es un trabajo decente y nadie debería mirarlas en menos. Estos elementos ayudaron a superar el sentido común de su posición subalterna (Gramsci, 1996). Ya no es normal o aceptable para ellas ser tratadas como la basura que limpian.

$\mathrm{Si}$ bien estas movilizaciones no terminaron con su subalternidad, en tanto no alcanzaron una autonomía total ni se convirtieron en el grupo social dominante (Gramsci, 1996), sí mejoraron algunas de sus condiciones laborales (ej. se sienten respetadas, pararon los abusos, la empresa las provee de más y mejores materiales, la universidad las incluye en algunos de los beneficios que entrega y hace unos meses comprometió un proceso de internalización) y eso ha tenido un impacto positivo en sus vidas y en su subjetividad. Las trabajadoras se sienten responsables de las mejoras en las condiciones laborales de todas las empresas de la universidad y se sienten orgullosas de sus logros. Más aún, a través de sus movilizaciones y su sindicalización, estas trabajadoras subalternas, como otras a nivel nacional (Rigat-Pflaum, 2008; Cid, 2012; Riquelme\& Abarca, 2015), desafiaron la idea de sujeto estándar de la acción colectiva, a saber, hombre y en empleos seguros.

\section{Conclusión}

La academia ha considerado prácticamente imposible que las trabajadoras subcontratadas de aseo se organicen, principalmente por sus condiciones de trabajo y el impacto del neoliberalismo en el sindicalismo. No obstante, tal como sucede en otros contextos, estas trabajadoras sí se movilizan. En ese sentido, más que no poder hacerlo, pareciera pasar que no se ha prestado atención a sus formas de organización.

En este artículo planteo que las trabajadoras subcontratadas de aseo en una universidad privada en Chile han sido construidas como sujetos subalternos, es decir, marginalizados y oprimidos (Gramsci, 1996), mediante una intersección de estructuras opresivas de género, clase y raza combinadas con las condiciones precarias de un trabajo subcontratado realizado en el marco de políticas y una gubernamentalidad neoliberal precarizadora. Estos elementos contribuyen a su marginalización dentro de la universidad y en la sociedad en general, fomentan su disgregación y dificultan sus posibilidades de organización.

No obstante, y a pesar de ello, un grupo de trabajadoras subcontratadas de aseo de una universidad privada se unió a un sindicato interempresa, se movilizó y enfrentó un proceso de huelga laboral para acabar con los abusos y las condiciones adversas de su trabajo. En este artículo planteo que fueron precisamente elementos de su subalternidad los que fomentaron su sindicalización. Así, este proceso se desencadenó principalmente para expulsar a una supervisora abusiva. En este sentido, su motivación se caracteriza por su espontaneidad (Gramsci, 1996). Sin embargo, también fue posible porque se involucraron en formas de sindicalismo comunitario. Las trabajadoras de aseo 
subcontratadas construyeron alianzas fuertes con estudiantes de la universidad y se unieron a un sindicato interempresa nacional. Estas alianzas, las características del liderazgo de su delegada sindical y la autoapreciación de su trabajo las empoderaron y las ayudaron a superar los miedos que las paralizaban.

Desde la mirada de la subalternidad y realizando entrevistas a estas trabajadoras, este artículo contribuye a investigaciones que se enfocan en las movilizaciones y organización de este tipo de trabajadores en otros contextos y desde otras perspectivas. Además, contribuye a la visibilización de un grupo y formas de movilización que no han sido consideradas por la academia chilena. No obstante, esta investigación requiere más desarrollo. Un vacío importante es la falta de datos relativos a la presencia de mujeres con ascendencia indígena y migrantes en este tipo de trabajo y las condiciones en las que lo desarrollan. Como mencioné a lo largo del artículo, estos datos son fundamentales considerando que se trata de un trabajo altamente marcado por dimensiones de género, raza y clase, y que las tasas de migración están aumentando en el país (Departamento de Extranjería y Migración, 2018). No saber en qué condiciones trabajan estas mujeres 0 cuántas son contratadas para realizar estos servicios, no solo invisibiliza estos grupos, sino que contribuye a su opresión y dificulta toda posibilidad de superar la subalternidad de las trabajadoras subcontratadas de aseo. Finalmente, es relevante expandir la investigación para incorporar las voces de otros actores relevantes en el proceso de sindicalización y movilización y profundizar en la información contenida en los archivos tanto del sindicato como de la agrupación de estudiantes que los acompaña. Esto permitiría expandir la historia de este grupo subalterno y desarrollar mejores estrategias para la superación total de su subalternidad (Gramsci, 1996).

\section{Bibliografía}

Abarzúa, Eduardo. (2008): "Acción sindical de trabajadores subcontratistas", en Soto y Gómez (Eds.) Flexibilidad laboral y subjetividades: hacia una comprensión psicosocial del empleo contemporáneo, p. 79-88. Santiago, Chile: LOM Ediciones.

Acciari, Luisa (2018): "Paradoxes of subaltern politics: Brazilian domestic workers' mobilisations to become workers and decolonize labour." (Tesis Doctorado) Londres, Reino Unido: London School of Economics and Political Science.

Aguiar, Luis (2004): "Resisting Neoliberalism in Vancouver: An Uphill Struggle for Cleaners." En Social Justice, Vol. 31, No. 3, p.105-129. 
Alberti, Gabriella (2016): "Mobilizing and Bargaining at the Edge of Informality: The "3 Cosas Campaign" by Outsourced Migrant Workers at the University of London." EnWorkingUSA, Vol. 19, No. 1, p.81-103.

Alcoff, Linda (1995): "The Problem of Speaking for Others" En J. Roof \& R. Wiegman (Eds.) Who can speak?: Authority and Critical Identity, p. 97-119. Urbana: University of Illinois Press.

Antileo, Enrique (2015): "Trabajo racializado. Una reflexión a partir de datos de población indígena y testimonios de la migración y residencia mapuche en Santiago de Chile". En Meridional. Revista Chilena de Estudios Latinoamericanos, p.71-96.

Bayat, Asef (2000): “From 'Dangerous Classes' to 'Quiet Rebels': Politics of the Urban Subaltern in the Global South." En International Sociology, Vol. 15, No. 3, p.533-557.

Boccardo, G., Cornejo, R., Gómz-Rubio, C., Vidal, P. \& Zavala-Villalón, G. (2015): Trabajo subcontratado en FCFM y FACSO. [online]. Disponible en https://www.researchgate.net/publication/305724776 Trabajo subcontratado en FCFM y FACSO

Boccardo, G., Moya, C., Ratto, N., Ruiz, F. (2016): Trabajo subcontratado en el área de aseo en la Pontificia Universidad Católica de Chile. Informe de Resultados. [online].

Disponible en https://archivosubcontrato.files.wordpress.com/2016/10/informe-final-13-deseptiembre-2016-definitivo.pdf

Boccardo, G., Ruiz, C., Cornejo, R., Bustamante, A., Martin, A., Becker, I. (2018): Caracterización de riesgos psicosociales en Trabajadoras de Casa Particular. [online]

Disponible en https://www.researchgate.net/publication/324455207 Caracterizacion de ries gos psicosociales en Trabajadoras de Casa Particular

Brown, Wendy (2015): Undoing the Demos: Neoliberalism's Stealth Revolution. New York: Zone Books.

Calderón, Ricardo. (2008): “Nuevas expresiones de acción sindical desde la precarización del empleo: El caso de los trabajadores contratistas de la gran minería del cobre", en Soto y Gómez (Eds.) Flexibilidad laboral y subjetividades: hacia una comprensión psicosocial del empleo contemporáneo, p. 111-133. Santiago, Chile: LOM Ediciones. 
CASEN. (2016): Inmigrantes. Principales resultados. [online]Disponible enhttp://observatorio.ministeriodesarrollosocial.gob.cl/casenmultidimensional/casen/docs/CASEN 2015 INMIGRANTES 21122016 EXTE NDIDA publicada.pdf

Cid, B. (2012): "Maternizando lo político: mujeres y género en el Movimiento Sindical de la Industria Salmonera Chilena." En Estudios Feministas, Vol. 20, No. 1, p.189207.

Cooper, B. (2016): "Intersectionality."En L. J. Disch\& M. E. Hawkesworth (Eds.) The Oxford handbook of feminist theory, p. 386-406. New York, NY: Oxford University Press.

Chile. Ministerio del trabajo y previsión social; subsecretaría del trabajo (16 de octubre del 2006). Ley 20123, Código del Trabajo. Artículo 183-A.

Crenshaw, Kimberle (1989): "Demarginalizing the Intersection of Race and Sex: A Black Feminist Critique of Antidiscrimination Doctrine, Feminist Theory and Antiracist Politics", en University of Chicago Legal Forum, p. 139-167.

Defensoría Popular de las y los Trabajadores. (2018): Posten Facebook [online]. Disponible enhttps://www.facebook.com/defensoriadelasylostrabajadores/posts/35688780 $\underline{1443725}$

De la Garza, Enrique. (2008): "La subcontratación y la acumulación de capital en el nivel global", en Celis (Ed.) La subcontratación laboral en América Latina: Miradas multidimensionales, p. 17-37. Medellín: Ediciones Escuela Nacional Sindical.

Departamento de Extranjería y Migración (2018): Estadísticas Migratorias. Registros administrativos del Departamento de Extranjería y Migración [online]. Disponible en https://www.extranjeria.gob.cl/estadisticas-migratorias/

Downey, Miguel \& Portiño, José Ignacio (2017): "Análisis del subcontrato en las universidades chilenas, especialmente en el caso de las universidades estatales y las vías jurídicas para su término". En Revista Chilena de Derecho del Trabajo y de la Seguridad Social, Vol. 8, No. 16, p. 81-100.

Duffy, Mignon (2007): "Doing the Dirty Work: Gender, Race, and Reproductive Labor in Historical Perspective". En Gender and Society, Vol. 21, No. 3, p.313-336. 
Revista Punto Género N.ㅇ 15 Junio de 2021

ISSN 0719-0417 / 114- 136

Echeverría, Magdalena (2010): La historia inconclusa de la subcontratación y el relato de los trabajadores. Santiago, Chile: División de Estudios, Dirección del Trabajo.

Echeverría, Magdalena (2013): "Subcontratos. La 'vía chilena' a la flexibilidad laboral y el nuevo sindicalismo". En J. Ossandón \& E. Tironi (Eds.), ADAPTACIÓN. La empresa chilena después de Friedman. Santiago, Chile: Ediciones UDP.

Fraser, Nancy (2016): "Contradictions of capital and care". En New Left Review, Vol. 100, p.99-117.

Fundación Sol (2014): LA SUBCONTRATACIÓN EN USACH. Una investigación cuantitativa para el proyecto de diagnóstico de las condiciones laborales de los trabajadores subcontratados de aseo, jardines y vigilancia de la Universidad. [online] Disponible en https://archivosubcontrato.wordpress.com/estudios-sobre-subcontrato/

Gramsci, Antonio (1996): Prison Notebooks (J. A. Buttigieg, Trans. Vol. II): New York: Columbia University Press.

Green, Marcus (2002): "Gramsci Cannot Speak: Presentations and Interpretations of Gramsci's Concept of the Subaltern." En A Journal of Economics, Culture \& Society, Vol. 14, No. 3, p.1-24.

Harvey, David (2007a): A brief history of neoliberalism. Oxford: Oxford University Press.

Harvey, David (2007b): "Neoliberalism as Creative Destruction". En The Annals of the American Academy of Political and Social Science, No. 610, p.22-44.

Hearn, Julie \& Bergos, Monica (2011): "Latin American cleaners fight for survival: lessons for migrant activism". En Race \& Class, Vol. 53, No. 1, p.65-82.

Hernández, R., Fernández, C. \& Baptista, P. (2010): Metodología de la investigación (5a ed.): México: McGraw-Hill.

Herod, Andrew \& Aguiar, Luis (2006a): "Introduction: Cleaners and the Dirty Work of Neoliberalism". En Antipode, Vol. 38, No. 3, p.425-434.

Herod, Andrew\& Aguiar, Luis (2006b): "Introduction: Cleaners' Agency". En Antipode, Vol. 38, No. 3, p.603-607.

Iranzo, Consuelo \& Richter, Jacqueline (2012): "Las implicaciones de la subcontratación laboral". En J. C. Celis (Ed.), La subcontratación laboral en América Latina: Miradas multidimensionales, p. 39-65. Medellín: Ediciones Escuela Nacional Sindical. 
Kilomba, Grada (2013): "Who Can Speak? Speaking at the Center, Decolonizing Knowledge". En G. Kilomba (Ed.) Plantation memories: episodes of everyday racism (3rd edition. ed.): Münster: Unrast.

Liguori, Guido (2015): "Conceptions of Subalternity in Gramsci". En M. McNally (Ed.) Antonio Gramsci, p. 118-133. London: Palgrave Macmillan UK.

Lugones, María (2007): "Heterosexualism and the Colonial/Modern Gender System". EnHypatia, Vol. 22, No. 1, p.186-219.

Madhok, Sumi., \& Evans, Mary (2014): "Epistemology and marginality". En M. Evans, C. Hemmings, M. Henry, H. Johnstone, S. Madhok, A. The SAGE Handbook of Feminist Theory, p. 1-8. Londres, Reino Unido: SAGE Publications Ltd.

Modonesi, Massimo (2012): Subalternidad. Mexico: Universidad Autónoma de México.

Mohanty, Chandra Talpade (1991): "Third World women and the politics of feminism". En C. T. Mohanty, A. Russo, \& L. Torres (Eds.) Under Western Eyes: Feminist Scholarship and Colonial Discourses,p. 51-80. Bloomington: Indiana University Press.

Mullassery, Sree (2002): "Other-to-Other: The Act of Speaking". En LSE Gender Institute Working Papers, No. 2, p.61-71.

Nakano Glenn, Evelyn (1992): "From Servitude to Service Work: Historical Continuities in the Racial Division of Paid Reproductive Labor". En Signs, Vol. 18, No. 1,p.143.

Nilsen, AlfGunvald \& Roy, Srila (2015): New subaltern politics: reconceptualizing hegemony and resistance in contemporary India. New Delhi, India: Oxford University Press.

Passos, Rachel Gouveia \& Nogueira, Cláudia Mazzei (2018): "Outsourcing and the sociosexual and racial division of labor". En Revista Katálysis, Vol. 21, p.484503.

Pedwell, Carolyn (2002): "Seeing The Self in The 'Other' And The 'Other' In The Self: (Intersubjective) Reflexivity - A Methodology For Representing 'Others'." LSE Gender Institute Working Papers, No. 2,p.71-81.

Rich, Adriene (1986): "Notes Towards a Politics of Location." En Blood, Bread and Poetry: Selected Prose 1979-1985, p. 210-231. London: Virago. 
Rigat-Pflaum, Maria (2008): Los sindicatos tienen género, p. 1-8. Fundación Friedrich Ebert.

Riquelme, Verónica \& Abarca, Manuela (2015): Más mujeres en los sindicatos. Sectores con alta sindicalización femenina. Santiago, Chile: Departamento de Estudios de la Dirección del Trabajo.

Rowbotham, Sheila (2006): "Cleaners' Organizing in Britain from the 1970s: A Personal Account", en Antipode, Vol. 38, No. 3, p.608-625.

Ruiz, Carlos \& Boccardo, Giorgio (2014): Los chilenos bajo el neoliberalismo: clases y conflicto social. Santiago, Chile: El Desconcierto, Fundación Nodo XXI.

Segovia, J., Ramirez-Aguilar, F., \& Zapata-Sepúlveda, P. (2018): "Experiencias Laborales de Mujeres Migrantes Afrocolombianas en el Norte de Chile". En Interciencia, Vol. 43, No. 8, p.544-551.

Soni - Sinha, U., \& Yates, C. (2013) ''Dirty Work?' Gender, Race and the Union in Industrial Cleaning." En Gender, Work \& Organization, Vol. 20, No. 6, p.737752.

Spelman, E. V. (1990): Inessential woman: problems of exclusion in feminist thought: Women's Press. Boston.

Spivak, Gayatri (1988): "Can the Subaltern speak?" En C. Nelson \& L. Grossberg (Eds.), Marxism and the interpretation of culture, p. 66-111. Basingstoke: Macmillan Education.

Valdés, X., Godoy, C., \& Mendoza, A. (2017): "Acción colectiva y resistencia: asalariadas agrícolas en Chile frente a la precarización laboral." En Izquierdas, Vol. 35, p.167-198.

Van Vlijmen, J. (2019): "Being a cleaner in The Netherlands: coping with the dirty work stigma". En Facilities, Vol. 37, No. 5/6,p.280-291.

Wills, J. (2008): "Making Class Politics Possible: Organizing Contract Cleaners in London." En International Journal of Urban and Regional Research, Vol. 32, No. 2, p.305-323. 